\title{
Deep brain stimulation for hyperkinetic disorders
}

\author{
ERWIN B. MONTGOMERY JR., M.D. \\ Department of Neurology, National Primate Research Center, University of Wisconsin-Madison, \\ Wisconsin
}

\begin{abstract}
Deep brain stimulation (DBS) has a record of safety and efficacy for an expanding range of indications. Recently, the Food and Drug Administration provided approval through a Humanitarian Device Exemption for DBS of the globus pallidus internus and subthalamic nucleus for the treatment of dystonia. There is increasing clinical experience demonstrating that DBS is also effective for other hyperkinetic disorders such as chorea from a variety of causes. The selection criteria, intraoperative targeting, and the postoperative management of DBS for hyperkinetic disorders are discussed.
\end{abstract}

\section{KEY WORDS - deep brain stimulation • dystonia - chorea • hyperkinesia • dyskinesia}

Despite the fact that the mechanisms of therapeutic action of DBS are unknown, this modality has demonstrated efficacy for an expanding range of indications. These include hyperkinetic disorders such as chorea, levodopainduced dyskinesia, and dystonia ${ }^{19}$ from a variety of causes. This article is a review of the indications for DBS in hyperkinetic disorders, and of patient selection, targeting, and postoperative management. It is written from the perspective of a physician who deals with patients in need of help for whom currently available therapies have proven insufficient. Anecdotal experience (some of which is considerable) of marked improvement legitimizes the question of when physicians should make this therapy available. It is somewhat disingenuous to suggest that patients with rare or unusual hyperkinetic disorders should await randomized controlled studies that probably would never be done or would be completed in a time frame that would have little relevance to the patient. Consequently, physicians are forced to extrapolate from a diversity of experience to make reasoned judgments for their patients' sake.

\section{OVERVIEW}

I will not catalog the experiences reported in the literature; extensive reviews by Vercueil, et al. ${ }^{39}$ and others already exist. Rather, this article begins with their findings as givens, and I have attempted to synthesize a rational and

Abbreviations used in this paper: $\mathrm{DBS}=$ deep brain stimulation; FDA $=$ Food and Drug Administration; $\mathrm{GPi}=$ globus pallidus inter nus; IRB = Institutional Review Board; PD = Parkinson disease; STN = subthalamic nucleus.
I hope reasonable approach to the management of disease in those patients who elect to undergo DBS for hyperkinetic disorders. The efficacy of DBS for a wide variety of hyperkinetic disorders indicates that this modality provides treatment of symptoms related to a possible common pathophysiological mechanism rather than to the pathogenetic mechanisms specific to each disease (see Mechanism of Action of DBS and Pathophysiology of Hyperkinetic Disorders later in this article). For example, DBS of the GPi has been described as improving dystonia caused by Hallervorden-Spatz syndrome, ${ }^{38}$ postanoxic dystonia, ${ }^{13}$ posttraumatic dystonia, ${ }^{25}$ choreoathetotic cerebral palsy, ${ }^{36}$ tardive dystonia,${ }^{37}$ and hemiballismus, ${ }^{41}$ and has been applied to Huntington disease. ${ }^{9}$ Although it is the consensus that patients with secondary dystonias do not improve to the same degree as DYT1-positive patients, ${ }^{39}$ nevertheless those with secondary dystonias do respond and should be considered in their own right. The fact that DBS of the thalamus improves tremor in patients with essential tremor or PD better than in those with multiple sclerosis does not mean that thalamic DBS is not helpful in the latter group of patients. ${ }^{27}$ Such generalization must be tempered, however, by the fact that atypical parkinsonism does not respond to DBS as does idiopathic disease. Although a patient with chorea from neuroacanthocytosis improved after pallidotomy, ${ }^{12}$ another did not respond to DBS of the GPi. ${ }^{44}$

The dilemma that confronts physicians and surgeons is what evidence, in type and amount, is sufficient to warrant recommendation of DBS. This dilemma is compounded by the fact that the decision often is made for the physician, surgeon, and patient by the business and adminis- 
trative policies of insurers. Furthermore, the application and perhaps more importantly the misapplication of evidenced-based medicine can be used to rationalize policies that are otherwise unreasonable. ${ }^{29}$ There are significant economic as well as logistical reasons why not every disease will have its treatment subjected to randomized controlled studies.

It is recommended that if DBS is considered for hyperkinetic disorders, particularly in cases other than dystonia, the treatment should be conducted at centers in which there is considerable experience with DBS for other movement disorders. There are several reasons for this; these include the following: 1) the importance of continued publication of experience; 2 ) the need for data to be of high quality including objective and outcome measures considered standard in clinical trials; and 3) the need to mitigate the possibility that negative outcomes are more related to surgical and neurological inexperience than to the procedure itself. I hope that less experienced physicians and surgeons will see that these recommendations are in their own best interest as well as that of their patients. Establishing the effectiveness of DBS for nondystonic hyperkinetic disorders by careful and expert application will make it much easier in the future for those with less experience, both technically and in securing health insurance coverage.

Given the extensive experience that it is reasonably safe and efficacious in the face of the demonstrated failure of possible alternatives, DBS of the GPi and perhaps of the STN could be considered a symptom-specific treatment rather than a disease-specific one. Consequently, patients with any hyperkinetic syndrome could be candidates, with the primary decision based on the ratio of risk to symptomatic benefit. Additional factors that must be considered include the following: 1) alternative therapies; 2) comorbidities that would influence risk; and 3) the patient's ability to cooperate with the surgical procedures.

Assessing whether the benefit in terms of symptom reduction is sufficient to outweigh the risks associated with the operation is problematic. Often physicians essentially make the decision for the patient by not offering DBS or by discouraging the patient from undergoing the procedure based on the physician's conclusion that the symptoms are insufficient to warrant surgery. With respect to such a conclusion, the question arises as to the basis for the physician's estimation of the degree of severity. How can any physician fully appreciate the degree of psychological, emotional, sociological, and physical toll when these are so particular to each patient? Furthermore, the assumption behind the ideal of patient autonomy is that it is the patient's (or his or her representative's) prerogative to make the decision, and that the role of the physician is that of an educator. Nevertheless, no physician can or should be required to treat a patient in a manner inconsistent with the practitioner's best judgment.

\section{FACTORS}

\section{Patient Selection}

Dystonia. The efficacy of this treatment for a wide range of types of dystonia and DBS locations was reviewed by the FDA and the results are available at their website (http://www.fda.gov/cdrh/pdf2/H020007b.pdf); studies and case reports up to 2003 are included. In their analysis they found that, given the considerable experience with DBS for PD and essential tremor, which could be generalized to predict the risks to be expected in DBS for dystonia, and the results of case reports in 201 patients, there was sufficient evidence to conclude a reasonable risk/benefit ratio. Consequently, FDA approval was granted through a Humanitarian Device Exemption for DBS as a treatment for dystonia. There have been numerous subsequent reports. ${ }^{2,4,6,20-23,34,46,47}$

The decision regarding DBS must take into consideration other alternatives, particularly medical ones, because of the risk of serious and/or irreversible complications from surgery. ${ }^{7}$ Alternative treatments for dystonia include anticholinergic drugs such as trihexyphenidyl (Artane), ${ }^{16,35}$ although most adults are unable to tolerate the high doses required. If the most disabling symptoms are limited to a relatively small number of muscles, intramuscular injections of botulinum toxin can be very efficacious. Orally or intrathecally administered baclofen may be helpful, but excessive sedation is a considerable problem. There are rare cases in which patients with dystonia respond dramatically to levodopa.

The decision whether to recommend DBS or intrathecally administered baclofen is hampered by the lack of clinical trials allowing comparisons between the two procedures. Although intrathecal baclofen may not pose a risk of intracranial hemorrhage, there are significant risks associated with sedation that are due to the drug itself and to infection, and mechanical failures. Furthermore, the efficacy of intrathecal baclofen in general has not been as impressive as that of DBS. ${ }^{10,35}$ One case report demonstrated improvement with DBS of the GPi after failure of intrathecal baclofen. ${ }^{40}$

Special consideration must be given to patients referred for botulinum toxin failure. There are numerous reasons for this failure, some of which may be reversible. Successful intramuscular injections of botulinum toxin require careful targeting. Often, electromyographic recordings are necessary to localize the target. As with any skill, experience and practice are key elements and physicians' abilities may vary; often one physician can use this treatment successfully, whereas another may not. Neutralizing antibodies to the specific botulinum toxin serotypes may develop and the use of alternative serotypes may restore responsiveness. A neurologist with considerable experience should be the one to make the decision that a patient's disease is refractory to botulinum toxin treatment.

Another alternative that should be considered in the case of cervical dystonia is partial and selective denervation. This procedure requires great care in isolating the branches of the peripheral nerve that selectively innervate the muscles contributing to the dystonia. Although there is no risk of intracranial hemorrhage and the risk of infection is considerably less, selective denervation still poses considerable risks associated with excessive denervation, which can cause disabling weakness that may be irreversible. In addition, dystonia is a dynamic process in which the patterns of muscle involvement change over time. As the disease progresses, muscles other than those initially denervated may become active in the dystonia. This could require repeated surgery with markedly in- 
creased risks for irreversible weakness. Unfortunately, there are no randomized or controlled studies in which selective denervation is directly compared with DBS. Consequently, the physician is left to use his or her judgment, as is often the case in clinical medicine, and is forced to make the comparison him- or herself.

Other Hyperkinetic Disorders. Alternative therapies for other hyperkinetic disorders, such as chorea or hemiballismus, are more limited. Medications include agents that block dopamine receptors, such as the traditional or atypical neuroleptic drugs, or agents that deplete dopamine stores in presynaptic dopamine neurons. The latter agents include tetrabenazine, which is not available in the US.

Tardive dyskinesia, tardive dystonia, Sydenham chorea, and hemiballismus are fundamentally different from other hyperkinetic disorders by virtue of the possibility of spontaneous reversal. The use of DBS is indicated for these conditions only after enough time has elapsed that the chance for spontaneous remission is low. Hemiballismus, which is usually secondary to an infarct in the STN, most often resolves within weeks to months. The spontaneous remission rate for tardive dyskinesia is approximately one third per year until 5 years have passed. ${ }^{14}$ After that, the chance of spontaneous remission is very low. Tardive dystonia has a somewhat worse prognosis.

There are a number of other rarer causes of hyperkinetic syndromes that are reversible without resorting to surgery or to medications that suppress dopaminergic function. It is important that these conditions be considered in the differential diagnosis and excluded. These include the following: 1) chorea caused by pregnancy, hormones, or birth control pills; 2) involuntary movements caused by liver or renal dysfunction; 3) pseudoathetosis caused by sensory loss; 4) cerebral vasculitis such as systemic lupus erythematosus; 5) Wilson disease; and 6) drugs such as cocaine. $^{26}$

Special Case of Children. Dystonia, particularly the generalized form, often affects children. There are several factors that must be taken into account when considering DBS in children. First, continued growth of the skull could cause migration of the DBS lead, although by 7 years of age the head circumference is approximately at the 90th percentile. In addition, there may be continued body growth that could cause breakage of the extension. The possibility of continued head and body growth affects the choice of surgical procedure.

The child's ability to cooperate with surgery needs to be assessed. Children who are unable to cooperate and remain awake while being treated with local anesthetic agents may require general anesthesia, which makes neurophysiological mapping for lead placement more problematic. In particular, it would be difficult to evaluate behavioral-physiological correlations such as sensorimotor driving of neuronal responses in the GPi or STN or induction of phosphenes by microstimulation of the optic tract with DBS of the GPi. Children as young as 11 years of age have undergone awake surgery successfully after induction of local anesthesia (my unpublished observations).

Additional concerns relate to the postoperative risks associated with childhood, particularly rough play. Parents and children should be advised about the necessity of avoiding rough play, particularly contact sports. The timing of surgery is important: earlier surgery may reduce the psychosocial complications that are particularly acute in adolescents and avoid orthopedic complications associated with chronic dystonia.

\section{Targeting of $\mathrm{DBS}$}

The GPi is the usual target in the treatment of hyperkinetic syndromes; however, there is growing interest in the STN as a target. Use of DBS in the GPi is remarkably effective. In the case of levodopa-induced dyskinesia, DBS of the GPi markedly reduces symptoms. Nonetheless, DBS of the STN is also effective in reducing levodopainduced dyskinesia, although the presumed mechanism has been the marked reduction in medications. Acutely, DBS of the STN may exacerbate levodopa-induced dyskinesia. Recent experience has shown that DBS of the STN can directly suppress dyskinesia as well. In our experience, STN stimulation at lower voltages can increase dyskinesia, which is suppressed at higher voltages. Although there is considerably less experience with DBS of the STN compared with that of the GPi, both are efficacious. There are technical advantages to DBS of the STN compared with that of the GPi. Target localization, both with neuroimaging and microelectrode mapping, is easier in the STN. ${ }^{3}$ Nonetheless, there is insufficient experience to recommend DBS of the STN over that of the GPi.

Targeting the GPi for DBS in dystonia is somewhat different from that in PD. This difference relates to the stimulation voltages, frequencies, and pulse widths required for dystonia. Some patients require high frequencies, voltages, and pulse widths. ${ }^{22}$ At these settings, the spread of electrical current can be much more extensive and the risks of side effects from inadvertent stimulation of surrounding structures is higher. Consequently, the DBS lead is placed farther from the posterior limb of the internal capsule and from the optic tract. The DBS lead is usually placed 3 to $4 \mathrm{~mm}$ from these structures. The precision necessary for the placement of the DBS lead mandates microelectrode recordings of neuronal extracellular action potentials for mapping.

Choice of DBS Leads. There are two commercially available DBS leads: one has four contacts that are 1.5 $\mathrm{mm}$ long and are separated by $1.5 \mathrm{~mm}$, for a total span of $10.5 \mathrm{~mm}$ (model 3387; Medtronic, Inc., Minneapolis, $\mathrm{MN})$. The second DBS lead has four contacts each 1.5 $\mathrm{mm}$ in length separated by $0.5 \mathrm{~mm}$, for a total span of 7.5 mm (model 3389; Medtronic, Inc.). There is very little reason to use the smaller-span DBS lead, particularly in the GPi, which probably requires stimulation of a larger volume. The smaller-span DBS lead was created specifically for the STN, but this was based on the presumption that stimulation of the STN is the therapeutic mechanism. There is considerable evidence, however, that the clinical efficacy of DBS of the STN probably has little to do with the stimulation of actual STN neurons but rather with the stimulation of the pallidofugal fibers that pass just above the STN. Clinical studies consistently have shown that the most effective stimulation sites are actually above the STN. ${ }^{42}$ Consequently, the larger-spaced DBS lead is probably more efficacious than the smaller-span leads.

Special Case of Children. The potential for continued 
growth and lead migration due to increased head size and extension fracture due to body growth need to be considered. In most cases of dystonia, the distal edge of the distal electrical contact is placed 3 to $4 \mathrm{~mm}$ above the optic tract or the identified bottom of the GPi. Nevertheless, in younger children the DBS lead could be placed somewhat deeper. For postoperative management of the disorder one would use the upper contacts initially and then the lower contacts as the patient's head size increases.

If two single-chamber impulse generators such as the Soletra (Medtronic, Inc.) are used, they need to be separated by at least 8 in to avoid cross-talk between stimulation times. Consideration should be given to placement of the impulse generator in the abdomen. In this case the longer extension $(95 \mathrm{~cm})$ might be considered. Alternatively, the recently approved dual-chamber impulse generator can be used (Kinetra; Medtronic, Inc.).

\section{Postoperative Management of Dystonia}

Postoperative management is complicated at best because of the large number of combinations of stimulation parameters. As yet, no efficient algorithm exists to explore the parameter space. Consequently, the only real guidelines, other than maximizing symptomatic control, are to reduce the drain on the battery and thus prolong its life. The factors that contribute most to battery drainage include levels above $3.7 \mathrm{~V}$ for the Soletra or above $3.2 \mathrm{~V}$ for the Kinetra device because of the voltage doubling circuit necessary to achieve stimulation voltages greater than that of the battery. Next in order of importance are large pulse widths, multiple active contacts, monopolar stimulation, and high frequency.

Programming for dystonia is further complicated by the time lag between stimulation adjustment and the improvement in symptoms, which may take weeks to months. Thus, it is very difficult to use the immediate symptomatic response in the clinic to assess the adequacy of the stimulation parameters; the physician needs to observe the patient for a sufficiently long period of time to assess the response. This means that an incremental approach starting from the most conservative stimulator settings may result in an inordinate amount of time is passing before symptomatic control achieved. Conversely, another approach is to initiate stimulation at the maximum parameters commonly found to be effective and tolerable. Once symptomatic control is achieved, aprogressive downward adjustment of the stimulation parameters can be undertaken to reach the minimum level sufficient to provide acceptable control.

The high voltage, frequencies, and pulse widths that may be required for dystonia increase the risk of excessive current being delivered to the brain. The standard accepted safety limit is 30 microcoulombs $/ \mathrm{cm}^{2} /$ phase. Unfortunately, commercially available stimulators provide a constant voltage and not a constant current. Consequently, it may be difficult to predict the amount of current being delivered to the brain. The amount of current sent into the brain is a function of the voltage and the impedance (a measure of resistance to the flow of electricity) of the DBS leads. The impedance may vary greatly between and within patients, particularly when there are multiple active contacts.
The programming device (model 8840 N'Vision Clinician Programmer; Medtronic, Inc.) provides a warning if the selected stimulation parameters are approaching the safety limit. Nonetheless, this is based on assumptions about the impedance of the DBS electrodes, which are based on the mean impedances found in PD clinical trials; these levels are on the order of $1000 \mathrm{ohms}$. The programming device bases its estimations on a conservative impedance value of $500 \mathrm{ohms}$. Although there is no reason to suspect that impedances for comparable settings are any different for patients with hyperkinetic disorders from ones for those with PD, for some patients the conservative estimate of $500 \mathrm{ohms}$ could result in a warning at settings that otherwise may be safe and effective. The impedance displayed on the "Therapy measurements" data screen of the programming device is a measure of the impedances specific to the active DBS lead contacts and the voltages and pulse widths for each patient. The programmer can consult nomograms (Fig. 1) that relate pulse width and voltage to the amount of current delivered at a given system impedance.

The impedance registered by the "Therapy measurements" is not the same as the "Electrode impedances." The latter calculates an impedance measure by using the factory settings for all pairs of contacts in the DBS lead. These values are used to test the integrity of the system and not to measure current delivered to the brain during therapeutic DBS.

\section{Mechanism of Action of DBS}

It is remarkable that despite the tremendous benefit of DBS for an expanding number of chronic neurological and psychiatric disorders, we have virtually no idea how this modality works. Unfortunately, this is a simple fact that some physicians have trouble expressing or accepting, and they often recite suppositions as though they were facts. Not only does this hinder research, it may convey a false sense of security to patients. Despite the similarity of clinical benefits between DBS and ablative procedures such as pallidotomy, it is a logical fallacy to infer that the underlying mechanisms are the same. There is considerable evidence that DBS activates rather than inhibits the stimulated target. ${ }^{31}$ How this increased activity improves hyperkinetic disorders is unknown.

Although these findings are based on uncontrolled case reports, the fact that hyperkinetic syndromes result from a wide range of disorders indicates that there may be a common mechanism that underlies hyperkinetic symptoms. Also striking is the number of different DBS sites that are effective. In addition to DBS of the GPi and STN, stimulation of the ventrolateral thalamus and motor cortex has been reported to be efficacious. For example, Katayama and colleagues ${ }^{17,18}$ reported that motor cortex stimulation could reduce posthemiplegic choreoathetosis, and Franzini, et al., ${ }^{11}$ and Canavero, et al., ${ }^{5}$ reported cases in which dystonia improved with motor cortex stimulation.

\section{Pathophysiology of Hyperkinetic Disorders}

The foregoing observations have important implications for the clinical use of DBS, for understanding the physiology of the basal ganglia and thalamocortical systems, and for understanding the pathophysiology of 


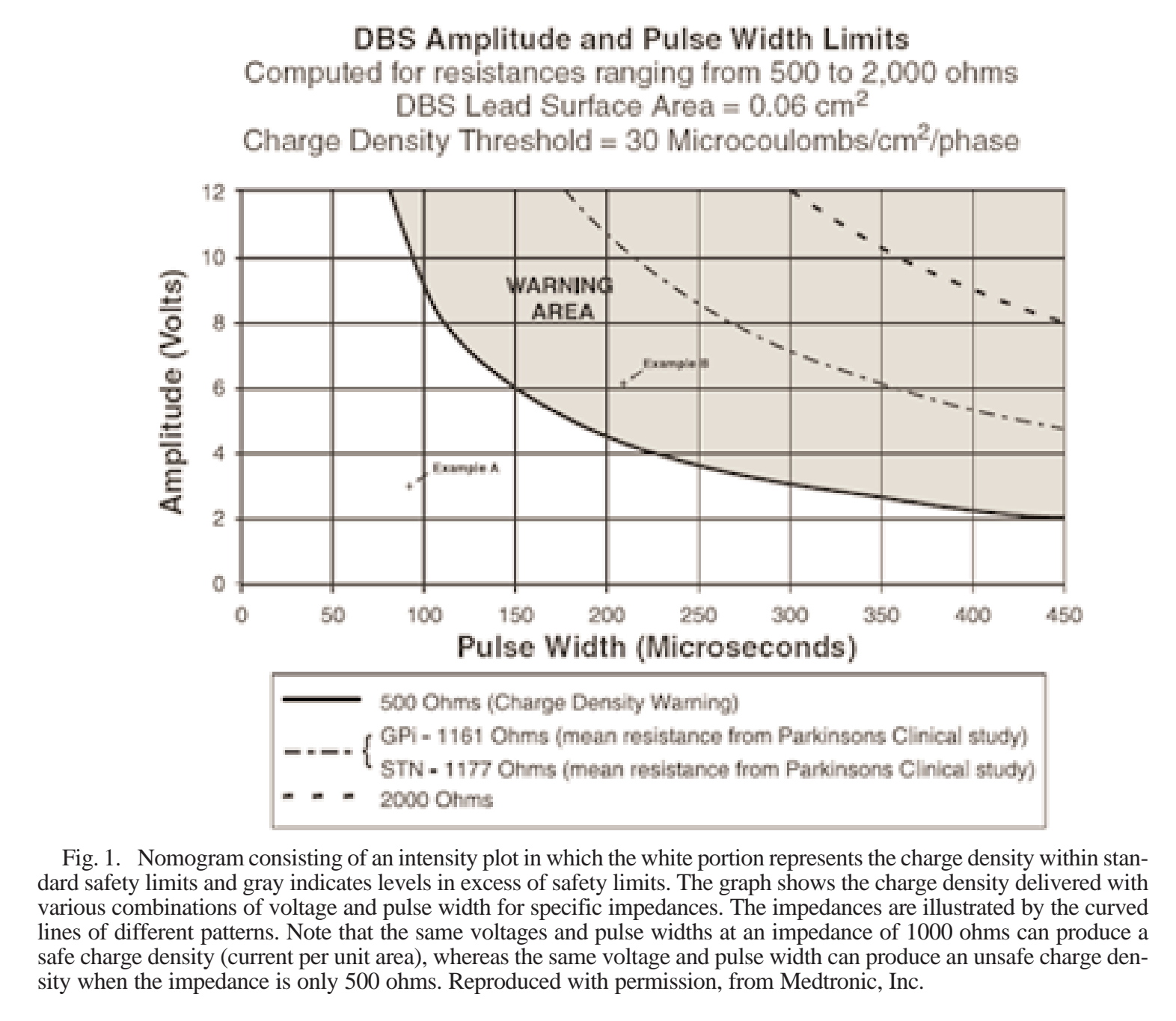

movement disorders. Clinically, the fact that a wide variety of hyperkinetic disorders respond to DBS indicates that this therapy is symptom-specific rather than diseasespecific. Consequently, the clinical experience with one type of hyperkinetic syndrome is relevant and generalizable to other types of hyperkinetic disorders. Thus, the large experience with DBS of both the GPi and the STN for treatment of levodopa-induced dyskinesia in PD provides strong support for the use of DBS in other hyperkinetic disorders. This has very important implications for health care coverage for DBS therapy in hyperkinetic disorders. Rather than having to provide evidence for the efficacy of DBS for specific diseases, which if rare means that the disease is likely to be "orphaned," it is reasonable to generalize from the collective experience.

The implications for the physiology and pathophysiology of the basal ganglia and thalamocortical systems are profound. The fact that lesions at multiple sites can have the same clinical presentation and the fact that DBS at multiple sites is effective argues strongly against the sequentially hierarchical schema of current theories. ${ }^{30}$ Rather, physiological functions, reflected by symptoms in dysfunction, are not localized but are represented in a distributed fashion throughout the basal ganglia and thalam- ocortical systems. Recently, a theory has been offered that describes the basal ganglia and thalamocortical systems as sets of dynamic, re-entrant, multineuronal, polysynaptic, nonlinear oscillators..$^{30}$

There are a number of theories about the mechanisms of hyperkinetic syndromes. Recordings from the GPi in patients with Huntington disease, dystonia, and levodopainduced dyskinesia show that the neuronal discharge activity may be at a lower frequency and more irregular. Use of DBS may increase the frequency of GPi neuronal discharge and make those patterns of discharge more regular. Thus, the therapeutic benefit may have less to do with the frequency of stimulation than with making the discharges more regular. Computational modeling indicates that low frequency and irregular activity may have a greater effect on neuronal information processing 22 through a process of stochastic resonance. This is the counterintuitive notion that adding noise to a signal can improve the signal-tonoise ratio. An analogy would be to add more noise to a radio signal that already has static, making it difficult to hear the music. Nevertheless, adding noise can increase the strength of the signal.

In the case of hyperkinetic syndromes, the lower and more irregular neuronal activity may exaggerate informa- 
tion being processed in the basal ganglia and thalamocortical system..$^{28}$ This exaggerated information is misinformation and could drive the abnormal involuntary movements. High-frequency DBS changes the low-frequency irregular activity to high-frequency, regular activity that has the least effect on information processing. Thus, exaggerated misinformation is changed to no information. In this limited sense, high-frequency, regular DBS is analogous to pallidotomy, which also removes misinformation.

Alternatively, Wu, et al. ${ }^{45}$ have suggested that DBS of the GPi inhibits local neurons by excitation of inhibitory presynaptic terminals; although this does not explain why motor cortex, thalamic, and STN stimulation also are effective in suppressing dyskinesia. Furthermore, there is evidence that although the cell bodies of neurons at the DBS site might be hyperpolarized and therefore less likely to initiate an action potential, the axon initiating segment may still generate action potentials in response to stimulation. $^{32}$

Perhaps the greatest obstacle to understanding the therapeutic mechanisms of DBS is the lack of understanding of the normal and abnormal physiology of the basal ganglia. The current theories ${ }^{1,8}$ posit a sequential, hierarchical organization in which information is processed through the striatum to the globus pallidus and intervening structures such as the STN and then relayed to the motor cortex through the ventrolateral thalamus. For the prototypical disorder, PD, investigators reason that loss of dopamine input ultimately results in excessive activity of the GPi, which inhibits the ventrolateral thalamus and decreases activity in the motor cortex. Similar notions have been applied to hyperkinetic disorders..$^{43}$ In this case, hyperkinetic disorders are associated with decrease activity in the GPi and subsequent decreased inhibition and increased activity of the ventrolateral thalamus. Nevertheless, such a hypothesis fails to explain the reduction of levodopa-induced dyskinesia after pallidotomy. ${ }^{24}$

Numerous criticisms of the current theory have been published..$^{28,31,32}$ The most telling evidence is that the differences in neuronal activities predicted by the sequential and hierarchical organization are not a necessary condition for disease. Hutchison, et al., ${ }^{15}$ have demonstrated that baseline GPi neuronal activity can be the same for patients with PD and those with Huntington disease. Similarly, experimental evidence showing no significant difference in the baseline neuronal activity of various structures in the basal ganglia and thalamocortical system in the primate model of parkinsonism induced by 1-methyl4-phenyl-2,5,6-tetrahydropyridine was published nearly 20 years ago. ${ }^{33}$

The current theories have considerable appeal in the almost mechanical nature of the intuitions. New theories will be orders of magnitude more complex. There is evidence, however, that the basal ganglia and thalamocortical systems behave in manners analogous to others that have been well described by the mathematics of complex systems. ${ }^{30}$ It is hoped that those mathematical intuitions can be translated into notions of basal ganglia physiology and pathophysiology. This will require a new vocabulary and a new understanding on the part of neurosurgeons and neurologists.

\section{Special Issue: IRB Approval and "Off-Label" Use}

The use of DBS for hyperkinetic disorders that are not approved by the FDA raises important issues in terms of liability and insurance reimbursement. This appears to be less of a problem for dystonia, given the FDA's Humanitarian Device Exemption. Nevertheless, the manufacturer of the approved device is required to be sure that centers in which DBS is performed for dystonia receive and maintain approval from their IRB before they provide devices specifically labeled for use in dystonia. Nevertheless, the same device is available without the specific dystonia labeling and such use would be considered "off-label."

This does not mean that such off-label uses are inappropriate, as would be the case for nondystonic hyperkinetic syndromes. Indeed, there are numerous instances in which off-label uses are considered standard care by experts. There is the danger that requiring patients to sign IRB consent forms would convey the wrong impression to both the patient and his or her insurer that DBS for dystonia is experimental or investigational.

In testimony before the Committee on Labor and Human Resources of the US Senate on February 22, 1996, Mr. William B. Shultz, Deputy Commissioner for Policy of the FDA, stated that "The Food and Drug Administration recognizes that, in certain circumstances, off-label uses of approved products are appropriate, rational, and accepted medical practice." He went on to state that "The legislative history of the Federal Food, Drug, and Cosmetic Act indicates that Congress did not intend FDA to interfere with the practice of medicine. Thus, once a drug is approved for marketing, FDA does not generally regulate how, or for what uses, physicians prescribed that drug. A physician may prescribe a drug for uses or in treatment regimens or patient populations that are not listed in the FDA-approved labeling."

Every day physicians use FDA-approved treatments for off-label uses as standard medical care. As noted in a letter by Dr. John Durant, Executive Vice President of the American Society of Clinical Oncology, to the acting commissioner of the FDA in 1998, “. . . more than half of all cancer patients receive anticancer drugs for off-label uses." He went on to state that "The most effective chemotherapy regimens are typically combinations of two or more approved drugs, yet combination regimens are not usually reviewed or approved by FDA. ..."

Recognizing the importance of many off-label treatments, the state of Ohio, as does many other states, has specific statutes requiring health insurance companies not to exclude off-label cancer treatments. The statute specifically states that "No individual or group health insuring corporation policy, contract, or agreement that provides coverage for prescription drugs shall limit or exclude coverage for any drug approved by the United States Food and Drug Administration on the basis that the drug has not been approved by the United States Food and Drug Administration for the treatment of the particular indication for which the drug has been prescribed...." The Ohio statutes then go on to provide criteria for approving such off-label treatments. These include "Two articles from major peer-reviewed professional medical journals [that] have recognized, based on scientific or medical criteria 
the drug's safety and effectiveness for the treatment of the indication for which it has been prescribed.?"

A reasonable conclusion would be that the lack of a specific labeled indication for an FDA-approved treatment or the lack of IRB approval is not and should not be a barrier to the use of treatment for the off-label diagnosis. Therefore, it is reasonable to conclude further that it is inappropriate for health insurance companies to define any off-label use or uses for which IRB approval is required as "experimental" and thus excluded from coverage.

\section{CONCLUSIONS}

The DBS of a variety of structures has been reported to improve hyperkinetic disorders such as dystonia and chorea arising from a variety of causes. In the absence of effective alternative medical therapies, DBS is relatively safe and effective. There is considerable evidence in the accumulated case studies that inspires confidence in DBS for dystonia. Although there are significantly fewer published cases of DBS for nondystonic hyperkinetic disorders, the frequency of positive outcomes argues that DBS should be considered symptomatic therapy rather than disease specific.

\section{References}

1. Albin RL, Young AB, Penney JB: The functional anatomy of basal ganglia disorders. Trends Neurosci 12:366-375, 1989

2. Albright AL: Neurosurgical treatment of spasticity and other pediatric movement disorders. J Child Neurol 18(Suppl 1): S67-S78, 2003

3. Baker KB, Boulis NM, Rezai AR, et al: Target Selection Using Microelectrode Recordings, Israel Z , Burchiel K (eds) in Microelectrode Recordings in Movement Disorders Surgery: New York: Thieme

4. Bronte-Stewart H. Surgical therapy for dystonia. Curr Neurol Neurosci Rep 3:296-305, 2003

5. Canavero S, Bonicalzi V, Paolotti R, et al: Therapeutic extradural cortical stimulation for movement disorders: a review. Neurol Res 25:118-122, 2003

6. Cif L, El Fertit H, Vayssiere N, et al: Treatment of dystonic syndromes by chronic electrical stimulation of the internal globus pallidus. J Neurosurg Sci 47:52-55, 2003

7. The Deep-Brain Stimulation for Parkinson's Disease Study Group. Deep-brain stimulation of the subthalamic nucleus or the pars interna of the globus pallidus in Parkinson's disease. $\mathbf{N}$ Eng J Med 345:956-963, 2001

8. DeLong MR. Primate models of movement disorders of basal ganglia origin. Trends Neurosci 13:281-285, 1990

9. Dostrovsky JO, Tang J, Lozano AM, et al: Alterted pattern of neuronal firing in pallidal neurons in Huntingon's and Parkinson's disease patients. Program no. 390.2, 2003 Abstract Viewer/Itineary Planner. Washington, DC: Society for Neuroscience, 2003, Online

10. Ford B, Greene PE, Louis ED, et al: Intrathecal baclofen in the treatment of dystonia. Adv Neurol 78:199-210, 1998

11. Franzini A, Ferroli P, Servello D, et al: Reversal of thalamic hand syndrome by long-term motor cortex stimulation. Case report. J Neurosurg 93:873-875, 2000

12. Fujimoto Y, Isozaki E, Yokochi F, et al: [A case of chorea-acanthocytosis successfully treated with posteroventral pallidotomy.] Rinsho Shinkeigaku 37:891-894, 1997 (Jpn)

13. Ghika J, Villemure JG, Miklossy J, et al: Postanoxic generalized dystonia improved by bilateral Voa thalamic deep brain stimulation. Neurology 58:311-313, 2002
14. Gotez CG, Horn S: Tardive dyskinesia, in Watts RL, Koller WC (eds): Movement Disorders: Neurological Principles and Practice. ed 2. New York: McGraw-Hill, 2004, pp 629-637

15. Hutchison WD, Lang AE, Dostrovsky JO, et al: Pallidal neuronal activity. implications for models of dystonia. Ann Neurol 53:480-488, 2003

16. Jankovic J: Treatment of dystonia, in Watts RL, Koller WC (eds): Movement Disorders: Neurological Principles and Practice, ed 2. New York: McGraw-Hill 2004, pp 527-540

17. Katayama Y, Fukaya C, Yamamoto T: Control of poststroke involuntary and voluntary movement disorders with deep brain or epidural cortical stimulation. Stereotact Funct Neurosurg 69:73-79, 1997

18. Katayama Y, Oshima H, Fukaya C, et al: Control of post-stroke movement disorders using chronic motor cortex stimulation. Acta Neurochir Suppl 79:89-92, 2002

19. Krauss JK, Loher TJ, Weigel R, et al: Chronic stimulation of the globus pallidus internus for treatment of non-dYT1 generalized dystonia and choreoathetosis: 2-year follow up. J Neurosurg 98:785-792, 2003

20. Krauss JK: Deep brain stimulation for dystonia in adults. Overview and developments. Stereotac Funct Neurosurg 78: 168-182, 2002

21. Kühn AA, Meyer BU, Trottenberg T, et al: Modulation of motor cortex excitability by pallidal stimulation in patients with severe dystonia. Neurology 60:768-774, 2003

22. Kupsch A, Klaffke S, Kuhn AA, et al: The effects of frequency in pallidal deep brain stimulation for primary dystonia. J Neurol 250:1201-1205, 2003

23. Kupsch A, Kuehn A, Klaffke S et al: : Deep brain stimulation in dystonia. J Neurol 250 (Suppl 1): I47-152, 2003

24. Lang AE, Lozano AM, Montgomery E, et al: Posteroventral medial pallidotomy in advanced Parkinson's disease. N Eng J Med 337:1036-1042, 1997

25. Loher TJ, Hasdemir MG, Burgunder JM, et al: Long-term follow-up study of chronic globus pallidus internus stimulation for posttraumatic hemidystonia. J Neurosurg 92:457-460, 2000

26. Mark MH: Other choreatic disorders, in Watts RL, Koller WC (eds): Movement Disorders: Neurological Principles and Practice. ed 2. New York: McGraw-Hill 2004, pp 639-655

27. Montgomery EB Jr, Baker KB, Kinkel RP, et al. Chronic thalamic stimulation for the tremor of multiple sclerosis. Neurology 53:625-628, 1999

28. Montgomery EB Jr: Neurophysiology/circuitry, Pahwa R, Lyons KE, Koller WC (eds),Handbook of Parkinson's Disease, ed 3. New York Marcel Dekker, 2003 pp 249-275,

29. Montgomery EB, Jr, Turkstra LS: Evidence-based practice: let's be reasonable. J Med Speech Lang Path 11: ix-xii, 2003

30. Montgomery EB, Jr, Dynamically coupled, high-frequency reentrant, non-linear oscillators embedded in scale-free basal ganglia-thalamic-cortical networks mediating function and deep brain stimulation effects. Nonlinear Studies (in press, 2004)

31. Montgomery EB Jr, Baker KB: Deep brain stimulation. Horch KW and Dhillon G (eds): Neuroprosthetics: theory and practice. Series on Bioengineering and Biomedical Engineering-Vol 2River Edge, NJ: World Scientific 2003 Chapter 65

32. Montgomery EB, Jr, Baker KB: Mechanisms of deep brain stimulation and future technical developments. Neurol Res 22: 259-266, 2000

33. Montgomery, EB, Jr, Buchholz SR, Delitto A, et al: Alterations in basal ganglia physiology following MPTP in monkeys, in Markey SP, Castagnoli N, Trevor AJ et al (eds) MPTP: A Neurotoxin Producing a Parkinsonian Syndrome Olando: Academic Press, 1986, pp. 679-682

34. Pastor-Gomez J, Hernando-Requejo V, Luengo-Dos Santos A, et al: [Treatment of a case of generalised dystonia using subthalamic stimulation.] Rev Neurol 37:529-531, 2003 (Spn)

35. Roubertie A, Echenne B, Cif L, et al: Treatment of early-onset 
dystonia: update and a new perspective. Childs Nerv Syst 16: 334-340, 2000

36. Thompson TP, Kondziolka D, Albright AL: Thalamic stimulation for choreiform movement disorders in children. Report of two cases. J Neurosurg 92:718-721, 2000

37. Trottenberg T, Paul G; Meissner W, et al: Pallidal and thalamic neurostimulation in severe tardive dystonia. J Neurol Neurosurg Psychiatry 70:557-559, 2001

38. Umemura A, Jaggi JL, Dolinskas CA, et al: Pallidal deep brain stimulation for longstanding severe generalized dystonia in Hallervorden-Spatz syndrome. Case report. J Neurosurg 100: 706-709, 2004

39. Vercueil L, Pollak P, Fraix V, et al: Deep brain stimulation in the treatment of severe dystonia J Neurol 248:695-670, 2001

40. Vesper J, Klostermann F, Funk T, et al: [Chronic high frequency deep brain stimulation of the globus pallidus internus for torsion dystonia.] Zentralblatt fur Neurochirurgie. 63:18-22, 2002

41. Vitek JL, Chockkan V, Zhang JY, et al: Neuronal activity in the basal ganglia in patients with generalized dystonia and hemiballismus. Ann Neurol 46:22-35, 1999

42. Voges J, Volkmann J, Allert N, et al: Bilateral high-frequency stimulation in the subthalamic nucleus for the treatment of Parkinson disease: correlation of therapeutic effect with anatomical electrode position. J Neurosurg 96:269-279, 2002
43. Wichmann T, DeLong MR. Functional and pathophysiological models of the basal ganglia. Cur Opin Neurobiol 6:751-758, 1996

44. Wihl G, Volkmann J, Allert N, et al: Deep brain stimulation of the internal pallidum did not improve chorea in a patient with neuroacanthocytosis. Mov Disord 16:572-575, 2001

45. Wu YR, Levy R, Ashby P, et al: Does stimulation of the GPi control dyskinesia by activating inhibitory axons? Mov Disord 16:208-216, 2001

46. Yianni J, Bain P, Giladi N, et al: Globus pallidus internus deep brain stimulation for dystonic conditions: a prospective audit. Mov Disord 18:436-442, 2003

47. Yianni J, Bain PG, Gregory RP, et al: Post-operative progress of dystonia patients following globus pallidus internus deep brain stimulation. Eur J Neurol 10:239-247, 2003

Manuscript received June 1, 2004.

Accepted in final form June 21, 2004.

Address reprint requests to: Erwin B. Montgomery, Jr., M.D., Department of Neurology, 5132, H6/538 CSC, University of Wisconsin-Madison, 600 Highland Avenue, Madison, Wisconsin 53792. email: montgomery@neurology.wisc.edu. 